Quantum Field Theory Under the Influence

of External Conditions (QFEXT11)

International Journal of Modern Physics: Conference Series

Vol. 14 (2012) 347-356

(C) World Scientific Publishing Company

DOI: $10.1142 / \mathrm{S} 2010194512007465$

\title{
FEYNMAN DIAGRAMS FOR DISPERSION INTERACTIONS OUT OF EQUILIBRIUM - TWO-BODY POTENTIALS FOR ATOMS WITH INITIAL EXCITATION
}

\author{
HARALD R. HAAKH ${ }^{1}$, JÜRGEN SCHIEFELE ${ }^{1,2}$ and CARSTEN HENKEL ${ }^{1}$ \\ ${ }^{1}$ Institut für Physik und Astronomie, Universität Potsdam, \\ Karl-Liebknecht-Str. 24/25, 14476 Potsdam, Germany \\ haakh@uni-potsdam.de \\ ${ }^{2}$ Departamento de Física de Materiales, \\ Universidad Complutense de Madrid, 28040 Madrid, Spain
}

Published 28 July 2012

\begin{abstract}
Diagrammatic techniques are well-known in the calculation of dispersion interactions between atoms or molecules. The multipolar coupling scheme combined with Feynman ordered diagrams significantly reduces the number of graphs compared to elementary stationary perturbation theory. We review calculations of van der Waals-Casimir-Polder forces, focusing on two atoms or molecules, one of which is excited. In this case, calculations of the corresponding force are notorious for mathematical issues connected with the spontaneous decay of the excitation. Treating such unstable states in a full non-equilibrium theory provides a physical interpretation of apparent contradictions in previous results and underlines the importance of decay processes for the intermolecular potential. This may have important implications on reactions in biological systems, where excited states may be relatively long-lived and the resonant intermolecular force may result in directed Brownian motion.
\end{abstract}

Keywords: van der Waals-Casimir-Polder interaction; Förster resonant energy transfer (FRET); non-equilibrium field theory

PACS numbers: 34.20.-b, 31.50.Df, 82.40.Bj

\section{Tools from Non-equilibrium Field Theory in Molecular QED}

The interaction between electrically neutral yet polarizable particles, commonly named after J. D. van der Waals, F. London, H. Casimir, and D. Polder, is one of the fundamental problems in atomic and molecular physics. While the two-body potential between two particles (atoms, molecules, nanoparticles) in their internal ground state is unambiguous, ${ }^{1-4}$ apparently incompatible results have been obtained if one of the atoms is prepared in an excited state. The quantity of interest is the longrange part of the potential that overwhelms the familiar van der Waals interaction: some calculations found this part to oscillate spatially, ${ }^{5-7}$ while later work found 
a monotonic power law. ${ }^{8-11}$ It is the aim of the present paper to understand these differences $^{9,10}$ better. We handle this non-equilibrium problem with a diagrammatic (Feynman graph) expansion.

The starting point of our quantum mechanical description is the electric dipole coupling Hamiltonian

$$
H_{A F}=-\sum_{n} E_{i}\left(x_{n}\right)\left[d_{i}^{n} \psi^{e}\left(x_{n}\right) \psi^{g \dagger}\left(x_{n}\right)+d_{i}^{n *} \psi^{g}\left(x_{n}\right) \psi^{e \dagger}\left(x_{n}\right)\right] .
$$

For simplicity, we treat the atoms as pointlike two-level objects located at the spacetime coordinates $x_{n}=\left\{\mathbf{r}_{n}, t_{n}\right\}$. The fermionic operators $\psi^{a \dagger}(x)$ create atoms in state $a=g$, e at $x$. Assuming that the transition dipoles $d_{i}^{n}$ are real, we work in the following with an effectively scalar electric field $E(x)=d_{i}^{n} E_{i}(x)$ whose space argument accounts for possible differences in the transition dipoles.

The textbook treatment of dispersive atom-atom interactions ${ }^{4,12}$ employs stationary fourth-order perturbation theory in $H_{A F}$. The summation over all possible intermediate states leads to a rather large number of terms. In this work, we discuss a more concise treatment using the closed time-path contour formalism due to Schwinger, Craig, Mills, and Keldysh (see Ref. 13). Following Sherkunov, ${ }^{10-11,14}$ the level shift of a ground-state atom is extracted from its full propagator

$$
i g_{\alpha \beta}^{(\mathrm{full})}(x, y) \equiv\left\langle T_{c}\left\{S_{2}(-\infty, \infty) S_{1}(\infty,-\infty) \psi_{\alpha}^{g}(x) \psi_{\beta}^{g^{\dagger}}(y)\right\}\right\rangle^{\mathrm{conn}}
$$

The Greek subscripts take the values 1 or 2 , denoting the branch of the Keldysh time contour which extends from $t=-\infty$ to $\infty$ (branch 1) and back (branch 2). The prescription $T_{c}\{\ldots\}$ orders operators according to their time arguments on the contour. Finally, $S$ is the standard scattering operator, the brackets $\langle\ldots\rangle$ denote an eigenstate of the non-interacting theory, and only connected products contribute. The Feynman formalism ${ }^{15,16}$ is valid for known in and out-states, which is the typical scenario in equilibrium systems at zero temperature. If, however, a general initial state (including, in our case, unstable excited atomic states) is prepared and then left to evolve under the interaction, the Keldysh formalism is necessary to describe all energetically allowed processes.

In the following, a propagator with both times on the forward branch will be used. Its series expansion is, from Eq. (2),

$$
\begin{aligned}
& i g_{11}^{(\text {full })}(x, y)=\left\langle T\left\{\psi^{g}(x) \psi^{g \dagger}(y)\right\}\right\rangle \\
& -\frac{1}{2} \int d t_{1} d t_{2}\left\langle T_{c}\left\{\sum_{\alpha, \beta=1}^{2}(-1)^{\alpha+\beta} H_{A F, \alpha}\left(t_{1}\right) H_{A F, \beta}\left(t_{2}\right) \psi_{1}^{g}(x) \psi_{1}^{g \dagger}(y)\right\}\right\rangle^{\text {conn }}+\ldots,
\end{aligned}
$$

where the first line is the ordinary (bare) Feynman propagator given in Eq. (A.1), with $T$ being the standard time ordering. We will see below that processes involving only ground-state atoms can be entirely described in terms of Feynman propagators.

The next section presents the calculation of self-energies for atoms and photons, and recovers the energy shifts and decay widths in a system of two particles. A comparison of the Feynman and Keldysh results suggests an interpretation of the 
apparent disagreement of earlier results: these may apply in different time regimes after preparation, separated by the time scale that characterizes energy transfer by an exchange of resonant photons. Finally, we discuss how resonant long-range potentials might influence some systems of biological relevance that are not commonly considered in the community present at this conference.

\section{Self-Energies with Dressed Photons}

Our perturbative analysis is built from two basic expressions: the self-energies of an atom coupled to the photon field and of a photon coupled to a (second) atom, respectively. Conceptually, this is closely related to the notion of dressed states. ${ }^{17}$ The real and imaginary parts of the self-energy

$$
\Sigma_{11}=\Delta E-i \frac{\Gamma}{2}
$$

can be identified as the energy-shift and the inverse lifetime (decay width) of the particle. We first calculate the atomic self-energy at next-to-leading order in the coupling to a generic photon field. In a second step, the interatomic interaction is identified by "dressing" the photons with a second atom. We only have to consider terms where photons are connecting the two atoms, provided we assume the transition frequencies to include the single-particle Lamb-shift.

\subsection{Single atom plus field: atom self-energy}

We start by considering the propagator of a ground-state atom $B$ (transition frequency $\omega^{B}$ ) at the one-loop level. Using the Feynman rules from the Appendices and App. C of Ref. 16, the evaluation of Eq. (3) yields

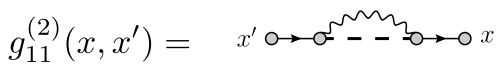

$$
\begin{aligned}
& =\int d^{3} x_{1} \int d^{3} x_{2} \int d \omega e^{-i \omega\left(t-t^{\prime}\right)} g_{11}\left(\omega, \mathbf{r}, \mathbf{r}_{1}\right) \Sigma_{11}^{g}\left(\omega, \mathbf{r}_{1}, \mathbf{r}_{2}\right) g_{11}\left(\omega, \mathbf{r}_{2}, \mathbf{r}^{\prime}\right)
\end{aligned}
$$

where the self-energy for a pointlike atom is to lowest order

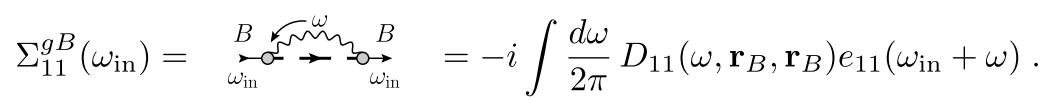

The excited-state propagator $e_{11}$ is given in Eq. (A.2) and $D_{11}$ denotes the Feynman propagator for photons. For the self-energy $\Sigma_{11}^{e}$ of an excited atom, replace $e_{11}$ by $g_{11}$ in the integral. This result can be brought into a more familiar form (see Ref. 18) by evaluating it on the mass shell, $\omega_{\text {in }}=\epsilon_{g}^{B}$, and writing the integral over positive frequencies only (recall that $D_{11}(\omega)$ is even in $\omega$ ):

$$
\Sigma_{11}^{g B}\left(\epsilon_{g}^{B}\right)=i \int_{0}^{\infty} \frac{d \omega}{2 \pi} D_{11}\left(\omega, \mathbf{r}_{B}, \mathbf{r}_{B}\right) \alpha_{11}^{g B}(\omega) .
$$


Here we recover the Feynman-ordered polarizability

$$
\alpha_{11}^{g B}(\omega)=\frac{1}{\omega^{B}-\omega-i 0^{+}}+\frac{1}{\omega^{B}+\omega-i 0^{+}},
$$

that is evaluated explicitly in Appendix A. (For the excited two-level atom $A^{*}$, replace $\omega^{B} \rightarrow-\omega^{A}$.) Note that the propagators here have poles in the upper left and lower right quadrant of the complex frequency plane (Feynman prescription). They coincide, however, with the usual retarded response functions at positive frequencies, and only these appear in the complex self-energy (7).

Substituting the bare photon propagator into $\Sigma_{11}^{g / e}$ yields, according to Eq. (4), an infinite energy shift which is, of course, the unrenormalized Lamb shift of atom $B$. The imaginary part of $\Sigma_{11}^{g}$ is nonzero if the temperature $T>0$ [see Eq. (B.1)], describing the absorption rate of thermal photons. At $T=0$, an imaginary part is found for the excited atom $A^{*}$ : this comes from a pole of $\alpha_{11}^{e A}(\omega)$ in the upper right quadrant and gives the spontaneous decay rate $\Gamma_{0}^{e A}=2 \operatorname{Im} D_{11}\left(\omega_{A}, \mathbf{r}_{A}, \mathbf{r}_{A}\right)$.

\subsection{Photon propagation in the presence of a second atom}

To evaluate the impact of a second atom (labeled $A$ ) on the self-energy of atom $B$, we take the diagram (6) and replace the photon propagator by its next-to-leading order correction:

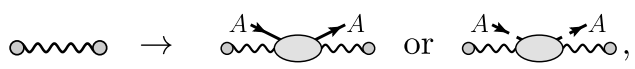

depending on the state of atom $A$. This "modular" strategy is, of course, consistent with the fourth-order expansion of the atom propagator $g_{11}$ in Eq. (2). The bare photon propagator $D_{11}\left(x, x^{\prime}\right)$ is given in Appendix $\mathrm{B}$, and its correction to leading order recovers Eq. (28) of Ref. 10

$D_{11}^{(2) a}\left(x^{\prime}, x\right)=\frac{-i}{2} \int d t_{1} d t_{2}\left\langle T_{c}\left\{\sum_{l, l^{\prime}=1}^{2}(-1)^{l+l^{\prime}} H_{A F, l}\left(t_{1}\right) H_{A F, l^{\prime}}\left(t_{2}\right) E_{1}\left(x^{\prime}\right) E_{1}(x)\right\}\right\rangle_{a}^{\text {conn }}$.

where $a=e, g$ denotes the state of atom $A$. For the purpose of our analysis it is sufficient to restrict the field to zero temperature which simplifies the treatment in the frequency domain considerably (see Appendix B). We find from Wick's theorem ${ }^{13}$

$$
\begin{aligned}
D_{11}^{(2) a}\left(\omega>0, \mathbf{r}^{\prime}, \mathbf{r}\right)= & D_{11}\left(\omega, \mathbf{r}^{\prime}, \mathbf{r}_{A}\right) \alpha_{11}^{a A}(\omega) D_{11}\left(\omega, \mathbf{r}_{A}, \mathbf{r}\right) \\
& -D_{11}\left(\omega, \mathbf{r}^{\prime}, \mathbf{r}_{A}\right) \alpha_{12}^{a A}(\omega) D_{21}\left(\omega, \mathbf{r}_{A}, \mathbf{r}\right)
\end{aligned}
$$

where the atomic polarizabilities are given in Appendix A. Note that this contains an extra term that is not in the form of Feynman propagators. There is no connected diagram involving $D_{22}$ and at positive frequencies, $D_{12}(\omega)=0$ (see Appendix B).

\subsection{Two ground-state atoms}

If atom $A$ is in the ground state $(a=g)$, the contribution from the second Keldysh branch vanishes because $\alpha_{12}^{g A}(\omega>0)=0$. We obtain a two-atom self-energy by 
substituting the dressed photon propagator $D_{11}^{(2) g}$ into $\Sigma_{11}^{g}$,

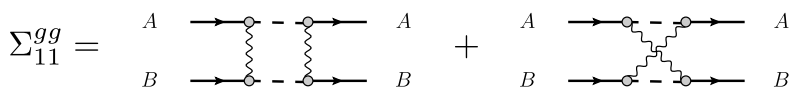

$$
\begin{aligned}
& =i \int_{0}^{\infty} \frac{d \omega}{2 \pi} \alpha_{11}^{g B}(\omega) \alpha_{11}^{g A}(\omega) D_{11}\left(\omega, \mathbf{r}_{A}, \mathbf{r}_{B}\right) D_{11}\left(\omega, \mathbf{r}_{B}, \mathbf{r}_{A}\right) \text {. }
\end{aligned}
$$

Only Feynman-ordered quantities contribute, and indeed, this situation corresponds to the ground state of the non-interacting theory where no non-equilibrium formalism is needed. The integration lends itself to a rotation to imaginary frequencies, and one sees that $\Sigma_{11}^{g g}=\Delta E^{g g}$ is a purely real two-body potential, equal to the well-known Casimir-Polder potential if the two atoms are embedded in free space. Note that the same calculation in traditional perturbation theory, e.g. in Ref.4, involves 12 diagrams with time-directed photon lines rather than the two Feynman diagrams above.

\subsection{Ground-state atom and excited atom}

The additional term in the photon propagator (9) makes the Feynman and Keldysh results differ. We find the following self-energy for the ground-state atom $B$ in the presence of an excited atom $A^{*}$

$$
\begin{aligned}
& \Sigma_{11}^{g e}=\quad i \int_{0}^{\infty} \frac{d \omega}{2 \pi} \alpha_{11}^{g B}(\omega)\left\{D_{11}\left(\omega, \mathbf{r}_{B}, \mathbf{r}_{A}\right) \alpha_{11}^{e A}(\omega) D_{11}\left(\omega, \mathbf{r}_{A}, \mathbf{r}_{B}\right)\right. \\
& \left.-D_{11}\left(\omega, \mathbf{r}_{B}, \mathbf{r}_{A}\right) \alpha_{12}^{e A}(\omega) D_{21}\left(\omega, \mathbf{r}_{A}, \mathbf{r}_{B}\right)\right\}
\end{aligned}
$$

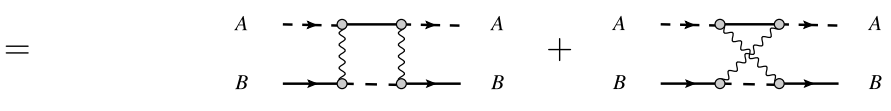

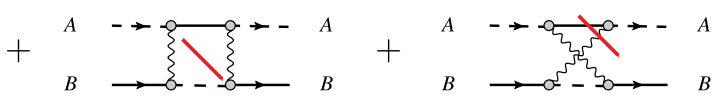

The first line is the result obtained in Refs. 5-7. In our formalism, this part of the result contains only time-ordered (Feynman) quantities and is represented by the first two diagrams of Eq. (11). We will argue in the next section under which conditions this line gives already the full result.

The second set of diagrams illustrates the terms that arise from the second Keldysh branch. There is one vertex beyond the (thick red) bars that corresponds to an interaction operator $H_{A F, 2}(x)$ on the second Keldysh branch. It will end leftmost after the action of the contour ordering operator $T_{c}$ and can be interpreted as acting directly on the outgoing states. This modification of the out-state translates the equilibration of the initial state (prepared at $t=-\infty$ and left to evolve under the interaction). The thick red bar thus illustrates the split between the branches of the Keldysh contour: it corresponds, apart from a prefactor, to cutting the diagrams and putting the loose ends on the mass-shell. The resulting two off-diagonal 
processes conserve energy (tree structure of the diagrams) and can be read as spontaneous emission from atom $B$ after a resonant photon exchange (left diagram) and as emission from atom $B$ stimulated by a photon from atom $A$ (right diagram).

To provide a more transparent physical interpretation, we bring the above result into a form involving retarded and advanced response functions. These are related to the Keldysh propagators by $\alpha_{11}=\alpha_{R}+\alpha_{12}$ and $D_{21}=D_{11}-D_{A}$ (see Refs. 13, 19). Remembering that for positive frequencies and $T=0$, we have $D_{11}(\omega)=D_{R}(\omega)$ and $\alpha_{11}^{g}(\omega)=\alpha_{R}^{g}(\omega)$, we get

$$
\begin{aligned}
\Sigma_{11}^{g e}=i \int_{0}^{\infty} \frac{d \omega}{2 \pi} \alpha_{R}^{g B}(\omega)\{ & D_{R}\left(\omega, \mathbf{r}_{B}, \mathbf{r}_{A}\right) \alpha_{R}^{e A}(\omega) D_{R}\left(\omega, \mathbf{r}_{A}, \mathbf{r}_{B}\right) \\
& +D_{R}\left(\omega, \mathbf{r}_{B}, \mathbf{r}_{A}\right) \alpha_{12}^{e A}(\omega) D_{R}\left(\omega, \mathbf{r}_{A}, \mathbf{r}_{B}\right) \\
& -D_{R}\left(\omega, \mathbf{r}_{B}, \mathbf{r}_{A}\right) \alpha_{12}^{e A}(\omega) D_{R}\left(\omega, \mathbf{r}_{A}, \mathbf{r}_{B}\right) \\
& \left.+D_{R}\left(\omega, \mathbf{r}_{B}, \mathbf{r}_{A}\right) \alpha_{12}^{e A}(\omega) D_{A}\left(\omega, \mathbf{r}_{A}, \mathbf{r}_{B}\right)\right\}
\end{aligned}
$$

The two lines indicated by the asterisk $(*)$ cancel out exactly. The first line gives an integrand regular in the upper right quadrant and can be rotated onto the imaginary axis; it does not yield any oscillating contributions. Within the two-level approximation, one has $\alpha_{R}^{e}=-\alpha_{R}^{g}$ [see Eq. (8)], so that the first term is equal, up to a global sign, to the ground state self-energy $\Sigma_{11}^{g g}$. The last line gives a purely resonant contribution because $\alpha_{12}^{e A}(\omega)=2 \pi i \delta\left(\omega-\omega_{A}\right)$ according to Eq. (A.6). It involves the modulus squared of the (retarded) photon Green function because $D_{A}(\omega)=D_{R}^{*}(\omega)$ at real frequencies. We thus rewrite Eq. (12) as

$$
\begin{aligned}
& \Sigma_{11}^{g e}=-\Sigma_{11}^{g g}-\alpha_{R}^{g B}\left(\omega_{A}\right)\left|D_{R}\left(\omega_{A}, \mathbf{r}_{B}, \mathbf{r}_{A}\right)\right|^{2}
\end{aligned}
$$

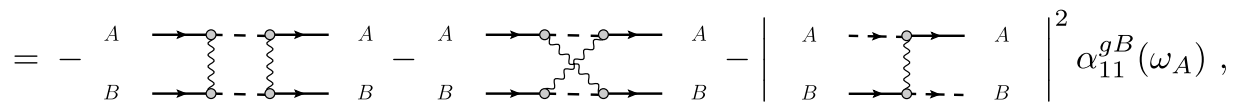

where the last term can be identified as Förster resonant energy transfer $2,12,20-23$ (FRET). Rather than distinguishing absorption and emission, the above reordering has led to a separation of dispersion (nonresonant or virtual photons) and FRET effects. The resonant energy exchange makes the ground-state atom $B$ unstable, and we recover the rate for FRET

$$
\Gamma_{\mathrm{FRET}}=-2 \operatorname{Im}\left[\Sigma_{11}^{g e}\right]=2 \pi \delta\left(\omega_{B}-\omega_{A}\right)\left|D_{R}\left(\omega_{A}, \mathbf{r}_{B}, \mathbf{r}_{A}\right)\right|^{2}
$$

in full agreement with the Golden Rule. The generalization of this rate to molecular emission and absorption spectra of finite width is straightforward and well-known. The FRET process comes along with a resonant two-body potential (the real part of $\Sigma_{11}^{g e}$ ) that has been discussed in Refs. 8-11. This potential does not oscillate spatially and lends itself to a simple semi-classical picture: the polarization energy of atom $B$ in the time-averaged field of a dipole source located at atom $A$, whose amplitude is fixed by the transition dipole. ${ }^{9}$ 


\subsection{Transient spatial oscillations of the resonant potential}

It is instructive to evaluate the contribution that was cancelled from Eq. (12) (upper line marked $(*))$. The explicit form of $\alpha_{12}^{e A}$ from Eq. (A.6) results in a spatially oscillating contribution to the self-energy

$$
\Delta \Sigma_{11}^{e g}=-\alpha^{g B}\left(\omega_{A}\right) D_{11}\left(\omega_{A}, \mathbf{r}_{B}, \mathbf{r}_{A}\right) D_{11}\left(\omega_{A}, \mathbf{r}_{A}, \mathbf{r}_{B}\right)
$$

Hence the first two (Feynman) diagrams in Eq. (10) give

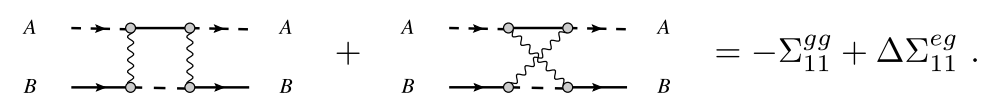

Since the potential $\Sigma_{11}^{g g}$ is monotonous, the extra term (14) is responsible for the spatially oscillating potential found in Refs. 5-7 using equilibrium (Feynman) theory. If all four diagrams are taken into account, one reaches a fully equilibrated state and the interaction $\Sigma_{11}^{e g}$ [Eq. (13)] does no longer contain any spatial oscillations. This is the result in Power and Thirunamachandran's calculations ${ }^{8,9}$ and differs from result for two ground state atoms due to the initial excitation energy $\hbar \omega_{A}$, which cannot be dissipated in the system.

We suggest the following scenario to understand the physical relevance of the two results, using the narrative of atom dressing. ${ }^{17}$ The interchange of virtual (or non-resonant) photons responsible for the monotonous part of the potential is very fast. This short-time behaviour is similar for both ground-state and excited atoms. The exchange of near-resonant photons takes a longer time due to the small frequency differences involved, and it leads to the equilibration of the initial state. This happens on the scale $1 / \Gamma_{\text {FRET }}$ set by the Förster rate. This time appears as a lower limit for the validity of the equilibrated potential obtained from $\Sigma_{11}^{e g}$. An upper limit is set by the spontaneous lifetime $1 / \Gamma_{0}^{e}$ of the atom $A^{*}$ when photons appear in free space modes. ${ }^{11,22}$ The two time scales are well-separated at short distance (non-retarded range, typical for FRET experiments).

The spatial oscillations of the potential (15) may therefore appear as a transient effect right after the preparation. We speculate that they remain visible if the excited state is continuously replenished with a sufficiently high rate (larger than $\Gamma_{\text {FRET }}$ ). This would require a time-dependent perturbative calculation similar to Ref. 2 that is beyond the scope of this paper. We note that a spatially oscillating dispersion interaction has been found at short times in the somewhat analogous situation of an excited molecule close to a surface. ${ }^{24}$

\section{Discussion and Conclusions}

The apparently differing predictions for the resonant part of the two-body potential between a ground-state and an excited atom have been a source of confusion for quite some time. We have reviewed this system in a non-equilibrium description (Keldysh closed time-path formalism) and identified how contributions that oscillate 
spatially disappear in such a description. The two results may correspond to different physical setups or to situations separated by a characteristic time scale, which we related to the rate of energy transfer (FRET): the oscillatory potential (Refs. 5-7) holding on very short time scales, and eventually evolving into the non-oscillatory one of Refs. 8-11.

In molecular physics, Förster broadening of atomic or molecular spectra is wellknown, but there seems to be less literature on the forces that come along with such resonant exchanges of energy. ${ }^{25}$ Actually, the term Förster force was coined as late as 2003 by Cohen and Mukamel. ${ }^{22,23}$ These forces may, however, play an important role in systems that stand in the focus of recent research, ranging from cold atoms ${ }^{26,27}$ over Rydberg states $^{28}$ to quantum dots, NV centers, and even biomolecules such as proteins and DNA. In the latter context, it has been proposed that diffusion-limited reactions between an excited and a ground-state reactant are facilitated by a state-selective force. The force would modify the motion of the molecules relative to undirected Brownian diffusion which may help to understand unusually high reaction rates. ${ }^{29}$ In biological systems in particular, the excited state may be pumped by an energy source (photoabsorption, chemical). Then the energy flow through the system will determine to what extent the scenario involving equilibration after excitation actually applies. Such questions certainly deserve further investigation, and underline that talking about unstable states as in dispersion interactions between atoms or molecules requires a careful characterization of the excited state in question.

We thank R. Behunin, S. Buhmann, G. Cammarata, F. Intravaia, V. Mkrtchian, R. Passante, G. Pieplow, J. Preto, S. Scheel, and S. Spagnolo for helpful discussions. Partial financial support by DFG, ESF network CASIMIR, GIF, and DAAD is acknowledged. All diagrams were created using Jaxodraw. ${ }^{30}$

\section{Appendix A. Atom fields and propagators}

Atoms are considered distinguishable by an index $n=A, B$, with an internal twolevel structure of energies $\epsilon_{g, e}^{n}$. The results will only depend on the Bohr frequencies $\omega^{n} \equiv \epsilon_{e}^{n}-\epsilon_{g}^{n}$. The annihilation operator $\psi_{a}^{n}(x)$ evolves in the interaction picture proportional to $\exp \left(-i \epsilon_{a}^{n} t\right)(a=g, e)$. Atoms are considered immobile and pointlike so that the position dependence is carried by the field operator in the interaction Hamiltonian (1).

The bare (leading order) propagator for a ground-state atom is given by

$$
g_{11}^{n}\left(x^{\prime}, x\right)=-i\left\langle\psi_{g}^{n}\left(x^{\prime}\right) \psi_{g}^{n \dagger}(x)\right\rangle \theta\left(t^{\prime}-t\right)=\int \frac{d \omega}{2 \pi} \frac{e^{-i \omega\left(t^{\prime}-t\right)}}{\omega-\epsilon_{g}^{n}+i 0^{+}} .
$$

For an excited atom, $e_{11}^{n}$ is obtained using $\epsilon_{e}^{n}$ in place of $\epsilon_{g}^{n}$. The step function $\theta\left(t^{\prime}-t\right)$ arises because in this non-relativistic theory, there are no anti-particles. In the frequency representation, we thus have the Feynman rules

$$
g_{11}(\omega)=\frac{1}{\omega-\epsilon_{g}+i 0^{+}}=\circ \_, \quad e_{11}(\omega)=\frac{1}{\omega-\epsilon_{e}+i 0^{+}}=\propto \rightarrow-\infty .
$$


We also need Keldysh propagators for the atomic polarization operator $P\left(x_{n}\right) \equiv$ $\psi^{e}\left(x_{n}\right) \psi^{g \dagger}\left(x_{n}\right)+\psi^{g}\left(x_{n}\right) \psi^{e \dagger}\left(x_{n}\right)$ that appears in the dipole interaction (1). The general correlation for atom $n$ in state $a$ and specifically in state $e$ are

$$
\begin{aligned}
\alpha_{\alpha \beta}^{a n}\left(x^{\prime}, x\right) & =i\left\langle T_{c}\left\{P_{\alpha}\left(x_{n}^{\prime}\right) P_{\beta}\left(x_{n}\right)\right\}\right\rangle_{a} \\
\alpha_{11}^{e n}\left(x^{\prime}, x\right) & =-\left(\left\langle e\left|\psi_{e}^{n \dagger}\left(x^{\prime}\right) \psi_{e}^{n}(x)\right| e\right\rangle g_{11}^{n}\left(x^{\prime}, x\right)+x^{\prime} \leftrightarrow x\right) \\
& =\int \frac{d \omega}{2 \pi} e^{-i \omega\left(t^{\prime}-t\right)}\left(\frac{-1}{\omega^{n}+\omega+i 0^{+}}+\frac{-1}{\omega^{n}-\omega+i 0^{+}}\right) .
\end{aligned}
$$

The Feynman polarizability $\alpha_{11}^{e n}(\omega)$ of the excited atom has a pole in the upper right quadrant of the $\omega$-plane which leads to a residue ("resonant contribution") when integration contours are shifted to the positive imaginary axis. The non-equilibrium polarizability gives only a resonant contribution

$$
\alpha_{12}^{e n}\left(x^{\prime}, x\right)=i\left\langle e\left|\psi_{e}^{n \dagger}(x) \psi_{g}^{n}(x) \psi_{e}^{n}\left(x^{\prime}\right) \psi_{g}^{n \dagger}\left(x^{\prime}\right)\right| e\right\rangle=\int \frac{d \omega}{2 \pi} e^{-i \omega\left(t^{\prime}-t\right)} 2 \pi i \delta\left(\omega-\omega^{n}\right) .
$$

To illustrate the link to the retarded polarizability, we form the combination

$$
\begin{aligned}
\alpha_{11}^{e n}\left(x^{\prime}, x\right)-\alpha_{12}^{e n}\left(x^{\prime}, x\right) & =-\int \frac{d \omega}{2 \pi} e^{-i \omega\left(t^{\prime}-t\right)}\left(\frac{1}{\omega^{n}+\omega+i 0^{+}}+\frac{1}{\omega^{n}-\omega-i 0^{+}}\right) \\
& =\int \frac{d \omega}{2 \pi} e^{-i \omega\left(t^{\prime}-t\right)} \alpha_{R}^{e}\left(\omega, \mathbf{r}_{1}, \mathbf{r}_{2}\right)
\end{aligned}
$$

which has poles in the lower half-plane only, as it should. For ground-state atoms, replace $\omega^{n} \leftrightarrow-\omega^{n}$ in these expressions, cf. Eq. (8) for $\alpha_{11}^{g n}$.

\section{Appendix B. Green functions of the free electromagnetic field}

We use the following Keldysh-Green functions for the photon field. To illustrate the formalism, we allow in this appendix for a thermal state with inverse temperature $\beta$ whence

$$
\begin{aligned}
& D_{11}\left(x^{\prime}, x\right)=i\left\langle T\left\{E\left(x^{\prime}\right) E(x)\right\}\right\rangle_{\beta}=\int \frac{d \omega}{2 \pi} e^{-i \omega\left(t^{\prime}-t\right)} D_{11}\left(\omega, \mathbf{r}^{\prime}, \mathbf{r}\right), \\
& D_{12}\left(x^{\prime}, x\right)=i\left\langle E(x) E\left(x^{\prime}\right)\right\rangle_{\beta}=i \int \frac{d \omega}{2 \pi} e^{-i \omega\left(t^{\prime}-t\right)} 2 \bar{n}(\omega) \operatorname{Im}\left[D_{R}\left(\omega, \mathbf{r}^{\prime}, \mathbf{r}\right)\right], \\
& D_{21}\left(x^{\prime}, x\right)=i\left\langle E\left(x^{\prime}\right) E(x)\right\rangle_{\beta}=-i \int \frac{d \omega}{2 \pi} e^{-i \omega\left(t^{\prime}-t\right)} 2 \bar{n}(-\omega) \operatorname{Im}\left[D_{R}\left(\omega, \mathbf{r}^{\prime}, \mathbf{r}\right)\right] .
\end{aligned}
$$

Note that the thermal occupation number becomes $n( \pm \omega) \rightarrow \mp \theta(\mp \omega)$ at zero temperature. The Fourier transforms $D_{12}(\omega)$ and $D_{21}(\omega)$ are then only supported by negative / positive frequencies, respectively. In the rest of the paper we always use this limit. The general case given above follows from the fluctuation-dissipation theorem. ${ }^{19,31}$ The link to the retarded and advanced Green functions is provided by the relations $D_{R}=D_{11}-D_{12}$ and $D_{\mathrm{A}}=D_{11}-D_{21}$. The Feynman propagator in the frequency domain is, therefore,

$$
D_{11}\left(\omega, \mathbf{r}^{\prime}, \mathbf{r}\right)=\text { anno }=\operatorname{Re}\left[D_{R}\left(\omega, \mathbf{r}^{\prime}, \mathbf{r}\right]+i \operatorname{coth}(\beta \omega / 2) \operatorname{Im}\left[D_{R}\left(\omega, \mathbf{r}^{\prime}, \mathbf{r}\right)\right]\right. \text {. }
$$


The retarded Green tensor in a linear and isotropic medium depends only on the difference $\mathbf{r}^{\prime}-\mathbf{r}$ and is given by

$$
D_{R}(\omega, \mathbf{r}, \mathbf{0})=\frac{\delta(\mathbf{r})}{3 \varepsilon_{0}} \mathbb{1}+\frac{\omega^{2}}{c^{2}} \frac{e^{i k r}}{4 \pi \varepsilon_{0} r}\left[\left(1+\frac{i}{k r}-\frac{1}{k^{2} r^{2}}\right) \mathbb{1}-\left(1+\frac{3 i}{k r}-\frac{3}{k^{2} r^{2}}\right) \hat{\mathbf{r}} \otimes \hat{\mathbf{r}}\right],
$$

where $\hat{\mathbf{r}}=\mathbf{r} /|r|$, and $k=\sqrt{\varepsilon(\omega) \mu(\omega)} \omega / c(\operatorname{Im}[k]>0)$ is the wave vector in the medium. Obviously, $\left[D_{R}(\omega, \mathbf{r}, \mathbf{0})\right]^{2}$ oscillates at half the medium wavelength, while $\left|D_{R}(\omega, \mathbf{r}, \mathbf{0})\right|^{2}$ does not. In the paper, $D_{R}\left(\omega, \mathbf{r}_{B}, \mathbf{r}_{A}\right)$ is evaluated by contracting the tensor above from left and right with the transition dipoles $\mathbf{d}^{B}$ and $\mathbf{d}^{A}$.

\section{References}

1. H. B. Casimir and D. Polder, Phys. Rev. 73, 360 (1948).

2. E. A. Power and T. Thirunamachandran, Phys. Rev. A 28, 2671 (1983).

3. A. McLachlan, Proc. Roy. Soc. Series A. 271, 387 (1963).

4. D. Craig and T. Thirunamachandran, Molecular quantum electrodynamics (Dover Publications, Mineola, New York, 1998).

5. R. R. McLone and E. A. Power, Proc. Roy. Soc. A. 286, 573 (1965).

6. M. R. Philpott, Proc. Phys. Soc. 87, 619 (1966).

7. G.-I. Kweon and N. M. Lawandy, Phys. Rev. A 47, 4513 (1993), erratum: Phys. Rev. A 49, 2205 (1994).

8. E. Power and T. Thirunamachandran, Phys. Rev. A 47, 2539 (1993).

9. E. Power and T. Thirunamachandran, Phys. Rev. A 51, 3660 (1995).

10. Y. Sherkunov, Phys. Rev. A 75, 012705 (2007).

11. Y. Sherkunov, Phys. Rev. A 79, 032101 (2009).

12. A. Salam, Molecular Quantum Electrodynamics (Wiley, Hoboken, New Jersey, 2009).

13. P. Danielewicz, Annals of Physics 152, 239 (1984).

14. Y. Sherkunov, Phys. Rev. A 72, 052703 (2005).

15. J. Schiefele and C. Henkel, Phys. Rev. A 82, 023605 (2010).

16. J. Schiefele, Ph.D. thesis, Universität Potsdam, 2011.

17. G. Compagno, R. Passante, and F. Persico, Atom-Field interactions and dressed atoms (Cambridge University Press, Cambridge, 1995).

18. J. M. Wylie and J. E. Sipe, Phys. Rev. A 32, 2030 (1985).

19. R. van Leeuwen, N. Dahlen, G. Stefanucci, C.-O. Almbladh, and U. von Barth, in Time-Dependent Density Functional Theory, Vol. 706 of Lecture Notes in Physics, edited by M. Marques et al. (Springer, Berlin, 2006), pp. 33-59.

20. T. Förster, Z. Naturforsch. A 4, 321 (1949).

21. D. Andrews, Chem. Phys. 135, 195 (1989).

22. A. E. Cohen and S. Mukamel, Phys. Rev. Lett. 91, 233202 (2003).

23. A. E. Cohen and S. Mukamel, J. Phys. Chem. A 107, 3633 (2003).

24. S. A. Ellingsen, S. Y. Buhmann, and S. Scheel, Phys. Rev. A 79, 052903 (2009).

25. V. M. Mal'nev and S. I. Pekar, Sov. Phys. JETP 31, 597 (1970).

26. W. R. Anderson, J. R. Veale, and T. F. Gallagher, Phys. Rev. Lett. 80, 249 (1998).

27. I. Mourachko, D. Comparat, F. de Tomasi, A. Fioretti, P. Nosbaum, V. M. Akulin, and P. Pillet, Phys. Rev. Lett. 80, 253 (1998).

28. J. A. Crosse, S. Å. Ellingsen, K. Clements, S. Y. Buhmann, and S. Scheel, Phys. Rev. A 82, 010901 (2010).

29. O. Berg and P. von Hippel, Ann. Rev. Biophys. Biophys. Chem. 14, 131 (1985).

30. D. Binosi, J. Collins, C. Kaufhold, and L. Theußl, Comp. Phys. Commun. 180, 1709 (2009).

31. G. S. Agarwal, Phys. Rev. A 11, 230 (1975). 